\title{
POLÍTICA MONETARIA, TASA DE INTERÉS Y DE DESCUENTO PARA EVALUAR PROYECTOS DE INVERSIÓN
}

\author{
MONETARY POLICY, INTEREST RATE AND \\ DISCOUNT TO EVALUATE INVESTMENT PROJECTS \\ Nicko Aib erto Gom ero Gonzaies * \\ Docente Principal de la Facultad de Ciencias Contables \\ Víctor Ricardo Masuda Toyofuku** \\ Docente Asociado de la Facuitad de Ciencias Contab ies \\ Universidad Nacional Mayor de San Marcos-UNMSM / Lima-Perú \\ [Recepción: Agosto 2016/ Conformidad: Setiembre 2016]
}

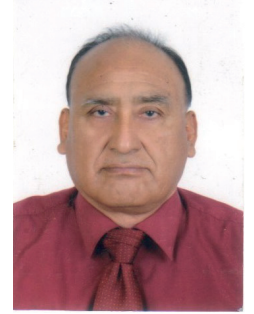

\section{RESUMEN}

Las inversiones privadas dependen de un conjunto de variables, entre las que están, el riesgo país y la tasa de interés; el grado de sensibilidad además dependerá de cómo se organice el mercado financiero, que en muchos casos, como el peruano presenta características oligopólicas, que en cierta forma generan un serie de distorsiones en el grado de influencia de las tasas de interés en las inversiones productivas.

Según la concepción teórica, la tasa de interés depende del tipo de política monetaria que ejecuten los bancos centrales, si esta es expansiva esta debe de caer, logrando un efecto contrario si la posición es restrictiva. Las evidencias señalan que el grado de correlación entre la emisión primaria y la tasa de interés es débil y positiva, por lo que los efectos que pueda lograr la autoridad monetaria es mínima con respecto a las inversiones. Evidenciando lo contrario con la tasa monetaria que ejerce elevada influencia en los tipos de interés y con ello a la tasa de descuento que se utiliza para evaluar inversiones.

\section{Palabras Clave:}

Inversiones, tasa de interés, tasa de descuento, rentabilidad, oligopolio

\begin{abstract}
Private investments depend on a set of variables, including country risk and interest rate. The degree of sensitivity will also depend on how the financial market is organized, which in many cases, such as the Peruvian, has oligopolistic characteristics. In a way, this may generate a series of distortions in the degree of interest rates influence on productive investments. According to the theoretical conception, interest rate depends on the type of monetary policy implemented by the central banks, if it is expansive it must fall, achieving an opposite effect if the position is restrictive. The evidence indicates that the degree of correlation between the primary issue and the interest rate is weak and positive, so that the effects that the monetary authority can achieve is minimal with respect to investments. The opposite is true of the monetary rate that exerts a high influence on interest rates and, therefore, the discount rate used to evaluate investments.
\end{abstract}

\section{Keywords:}

Investments, interest rate, discount rate, profitability, oligopoly

\footnotetext{
* Doctor en Ciencias Económicas. Magíster en Contabilidad con mención en Banca y Finanzas -UNMSM. Email: gomero_econ@yahoo.es

**Máster en Tributación y Política Fiscal. Email: vmasudato@gmail.com
} 


\section{INTRODUCCIÓN}

El crecimiento sostenido de una economía está supeditada a como se desarrollen las inversiones en el tiempo. Si los actores económicos que están ligados a los flujos de estos capitales observan escenarios favorables para realizar sus inversiones, las fortalezas macroeconómicas serán elocuentes, cuyos efectos se verán traducidos en una mejora importante del PBI. Es más, los impactos se extenderán hasta el mercado laboral en donde se crearan nuevos puestos de trabajo, pero de tipo estructural. Las bondades de las inversiones, incluyendo las estatales son importantes, que a través de los efectos multiplicadores, crean las condiciones del caso para fortalecer las posiciones económicas de un país.

En esta dinámica de impulsar las inversiones, juegan un papel importante las medidas monetarias que puedan diseñar e implementar los bancos centrales. Como la correlación entre la tasa de interés y las inversiones, es inversa, para impulsar las inversiones, la política monetaria debe tener características expansivas, logro que se lograría por cambios a la baja de los tipos de interés. La efectividad de los cambios de la tasa de interés no solo estará influenciado por las medidas monetarias, sino además por las características propias del mercado financiero.

La investigación apunta a evaluar, como la oferta monetaria a través del mecanismo de transmisión influye en la tasa de descuento, que posee como componente a la tasa activa, que se diferencia por cada tipo de sujeto prestatario. La rentabilidad de las inversiones, medida a través del VAN, es afectada por la tasa descuento llamada WACC (costo promedio ponderado del capital), utilizándose para la determinación la tasa de interés, que como ya se conoce se despeja en el mercado de intermediación indirecta. Esta relación de causalidad es el objetivo que pretende demostrar el presente estudio.

En el presente estudio se analiza; la oferta, la política monetaria, la base monetaria, la tasa de interés, la tasa de descuento. Valor Actual Neto de una inversión. Variables que son contrastadas para evidenciar las relaciones de causalidad y así lograr el objetivo del presente artículo de investigación

\section{Un enfoque macroeconómico de la política mo- netaria y la tasa de interés}

La política monetaria que es diseñada y aplicada por los Bancos Centrales no solo genera efectos en la estabilidad económica, sino, que además sus impactos, vía el mecanismo de transmisión, conocida como la tasa de interés, se constituye en una herramienta de primer nivel para dinamizar las inversiones provenientes del sector privado. Hay que destacar que este tipo de actividad también, lo podemos encontrar en el sector público, pero la diferencia radica, que el primero muestra sensibilidad a los tipos de interés y el segundo está supeditada a las decisiones políticas que provienen del Poder Central. Pero ambos generan efectos multiplicadores que van apalancar el crecimiento económico. Dornbush Fiseher ( 2015) en sus texto de macroeconomía explica que , “... al ejecutarse gastos de este tipo el PBI va a experimentar un crecimiento mayor a las inversiones ejecutadas, jugando un papel importante en este resultado el multiplicador, que a la vez e estará influenciada por la propensión al consumo y el tamaño del impuesto ...”

Como se conoce, una de las principales funciones de la autoridad monetaria es el control de la inflación y con ello mantener estable el valor de la moneda, pero también posee responsabilidad en aplicar políticas coordinadas con el responsable de la política fiscal para lograr mejores escenarios en el campo macro y microeconómico. Las medidas que aplique no solo podrían estar enfocadas a potencializar las inversiones sino que además los efectos pueden llegar a dinamizar el consumo de las familias, quienes, como se conoce, toman esta decisión en función como se comporte los tipos de interés. Por ello se argumenta y así bien lo evidencia Mankiw (2014) “...que los movimientos cíclicos de la demanda agregada va a estar influenciada por las medidas monetarias que ensaye la autoridad monetaria...". Es decir los efectos no solo se quedan en el sector monetario sino que trascienden el sector real de la economía, que es en donde se encuentran las unidades productivas o inversionistas, quienes son los artífices en la construcción de una economía sólida y sostenida.

Las teorías económicas y financieras sostienen que el flujo de inversiones de carácter interno o externo, no son sensibilizadas únicamente por los tipos de interés o costo de los créditos, sino que su dinámica 
está relacionada por un conjunto de factores catalogados como exógenos y que provienen del entorno económico, político y social que de mostrar signos de debilidad e incertidumbre elevaran el Riesgo País o EMBI que es considerada como variable crítica para atraer o ahuyentar las inversiones productivas o de largo plazo. Por ello es importante la calificación de riesgo que realizan a cada país las calificadoras como Moody's o Standard \& Poor's, cuyas conclusiones en cuanto al escenario económico representan una garantía de estabilidad para las inversiones o en caso contrario señalan volatilidades con efectos perniciosos en los flujos de capital.

Es preciso destacar que estas inversiones son las que fundamentan la formación de empleos estructurales, el pago de impuestos, el ingreso de divisas, fortalecimiento del PBI entre otras bondades, cuyos resultados son indicadores de fortaleza económica. No existe país que no haya fundamentado su crecimiento económico sin flujos sostenidos de inversiones provenientes del sector privado y complementariamente del Estado. No hay que perder de vista que estos últimos desembolsos se efectivizan gracias al pago de impuestos de los contribuyentes que es parte del ahorro interno, que de no cubrir las necesidades sociales son calzadas con deudas soberanas traducidas en la emisión de bonos soberanos. Al establecer una correlación entre las variables inversión y otros agregados macroeconómicos los estudios evidencian una elevada causalidad de tipo directa, lo cual es beneficioso para toda economía.

Hay que precisar que existen otras inversiones que muestran poca sensibilidad a los vaivenes de la tasa de interés, estas son consideradas de corto plazo o especulativos, cuyas rentabilidades están basadas en la diferencia de precios, márgenes que son obtenidos gracias a la volatilidad de los activos financieros que se negocian en el mercado de capitales o del dinero. El análisis fundamental y técnico se constituye en herramienta básica para tomar posiciones, para obtener óptimos resultados, esperados por la estructuración de los portafolios.

Si bien el mercado de capitales, especialmente el secundario, donde opera la Bolsa de valores, es utilizado por los cortoplacistas para optimizar la gerencia de su portafolio, este mercado no deja de ser una importante fuente para obtener recursos financieros, para financiar importantes alternativas de inversión. Hay que tener en cuenta que las corporaciones o negocios calificados utilizan esta vía para emitir instrumentos de deuda o capital que van a parar en las manos de inversionistas que estructuran carteras con estos instrumentos emitidos, pero como contrapartida entregan capitales frescos para que sean colocados por las empresas en actividades de inversión.

Hay que señalar que el grado de impacto de la tasa de interés va más allá de las inversiones, también se focaliza en la valorización de instrumento de deuda o de renta fija, cuya variabilidad de sus precios en el mercado secundario va a estar influenciada por la tasa de interés. Mejor dicho, el hecho que el precio del bono se ubique sobre, bajo o igual a la par, es porque va existir la influencia de la volatilidad de la tasa de interés, el cual como se argumentó es influenciada por el tipo de política monetaria. Afirmación que se aprecia en el texto de Ross (2012), quien explica claramente este tipo de causalidad.

Como se puede apreciar, la autoridad monetaria al condicionar el comportamiento de la tasa de interés no solo ejerce influencia para mantener la estabilidad económica sino que además puede dinamizar otros mercados, que a través de los efectos multiplicadores van a fortalecer los cimientos económicos de un país. Bajo este marco los Bancos Centrales no solo deben orientar sus políticas para mantener la inflación en los márgenes permitidos o en todo caso determinar la oscilación de los tipos de cambio, sino que sus medidas deben ir más allá del logro de estos objetivos macroeconómicos, como es la de potenciar las inversiones y las operaciones bursátiles. Claro está que, dentro de esta gestión monetaria no se tiene que perder el control de la demanda agregada ya que no debe llegar a la zona de sobrecalentamiento de la economía, por ello el protocolo económico es echar mano a las políticas contra cíclicas.

Por ultimo cabe precisar que los gestores privados, los que actúan en el mercado de bienes y financiero, deben seguir de cerca las posiciones de política económica del Banco Central, solo así estará en ventaja al tomar sus decisiones. Una pregunta que tendría que responder un prestatario sería: ipor qué 
no endeudarse con tasas variables y si con tasas fijas? $\mathrm{O}$ ¿por qué no tomar una posición contraria? o en todo caso, ¿̇por qué no asumir una deuda en divisa y si en moneda nacional? Su respuesta por cierto deberá estar sustentada bajo el marco de las políticas que puedan ensayar las autoridades monetarias.

\section{La política monetaria, la tasa de interés y las in- versiones. Caso del WACC y del COK. Enfoque financiero}

Para determinar la viabilidad económica y financiera de una inversión el Valor Actual Neto (VAN), la Tasa Interna de Retorno (TIR) y el coeficiente beneficio - costo se constituyen en herramientas básicas lograr tal propósito. Como se conoce en el mundo de las inversiones, ningún inversionista puede arriesgar fondos sin antes saber cuánto será el retorno esperado por tales desembolsos, por ello utiliza indicadores de rentabilidad, que según Sapag Nassir (2008) se puede determinar para el proyecto y para el capital.El primero de estos, determina la bondad del proyecto sin apalancamiento financiero, y el segundo plasma su evaluación considerando los flujos financieros cuyo resultado es de interés para los accionistas o de quienes están detrás de la inversión. Por ello Baca Urbina (2006), explica a través de sus teorías que estos resultados son básicos para determinar los tipos de rentabilidad de la inversión a realizar, y como se sabe, está influenciada por la tasa de interés y por ende por la tasa de descuento.

Si se desea saber la fortaleza de la inversión ante choques externos se tiene que someter a un proceso de sensibilización, es decir se tiene que elegir variables críticas o más representativas que afecten la rentabilidad de la inversión y alterarlos en forma intencionada. Sin duda una variable crítica es la tasa de descuento en cuya estructuración esta la tasa de interés.

Pero, ¿qué tiene que ver la política monetaria con las bondades de una inversión? Como se señaló en un principio, los Bancos Centrales pueden influir en el comportamiento de la tasa de interés vía el manejo de la oferta monetaria y así también lo explica la teoría macroeconómica como el que se encuentra en el texto de DORNBUCH FISHER (2010) quien llega explicar claramente la relación inversa que existe entre estas dos variables. Si se desea que baje los tipos de interés tiene que aumentar la oferta monetaria y si el objetivo es todo lo contrario se tiene que aplicar medidas restrictivas que conlleven a limitar la cantidad de liquidez en el sistema. Como se puede ver, la volatilidad de los tipos de interés va a depender del tipo de política que ensaye el Banco Central. Por ello, los textos básicos de economía señalan que si se quiere aumentar las inversiones se debe bajar los tipos de interés y si el propósito es realizar ajustes a la demanda agregada, la tasa de interés tiene que ir en dirección contraria, lo cual conllevará a contraer las decisiones de inversiones provenientes del sector privado.

Por otro lado cabe precisar, la tasa de interés se ajusta en el mercado monetario en donde participan las IFIS, las distorsiones que se puedan generar en esta variable va estar correlacionada a la estructura del mercado, si esta es oligopólica, como es la peruana, las tasas de interés aparte de las decisiones de la autoridad monetaria serán afectadas por el grado de concentración de los intermediarios financieros, instituciones que colocaran tasas de interés a las operaciones de créditos lejos de lo que dicte una libre y justa competencia.

Si se hace un contraste entre la tasa de interés y la Emisión Primaria, se confirma la afirmación anterior, ya que entre estas dos variables no existe una relación inversa tal como reza la teoría macroeconomía, sino todo lo contrario, se evidencia una correlación directa en su recorrido, sustentándose este hecho en el estadístico coeficiente correlación $(\mathrm{R}=0.06)$. El Resultado obtenido explica una débil dependencia de tipo directa entre estas dos variables, contradiciendo así lo afirmado por la teoría económica. La tasa de interés, al no responder en forma contraria a las señales de la oferta monetaria, es porque existen factores producto del oligopolio bancario que condicionan el comportamiento del costo financiero. Con lo cual queda evidenciada la poca eficacia de la autoridad monetaria con el manejo de la liquidez para condicionar el comportamiento de la tasa de interés. 
Tabla No 01

Emisión primaria y tasa de interés (var \%)

\begin{tabular}{|l|c|c|}
\hline \multicolumn{1}{|c|}{ MES } & EMISIÓN PRIMARIA & TASA DE INTERÉS \\
\hline Enero & -1.30 & 7.10 \\
\hline Febrero & -2.10 & 6.90 \\
\hline Marzo & 3.00 & 6.80 \\
\hline Abril & -2.60 & 6.70 \\
\hline Mayo & 0.40 & 6.60 \\
\hline Uunio & -1.10 & 6.60 \\
\hline Uulio & 4.60 & 6.60 \\
\hline Agosto & -2.40 & 6.90 \\
\hline Septiembre & -2.10 & 7.00 \\
\hline Octubre & -1.70 & 7.10 \\
\hline Noviembre & 0.10 & 7.20 \\
\hline Diciembre & -4.80 & 7.30 \\
\hline Enero & -1.70 & 7.30 \\
\hline Febrero & 0.80 & 7.40 \\
\hline Marzo & 0.90 & 7.50 \\
\hline Abril & 2.20 & 7.50 \\
\hline Mayo & 2.70 & 7.50 \\
\hline Uunio & 3.60 & 7.60 \\
\hline Uulio & 1.90 & 7.70 \\
\hline Agosto & 5.40 & 7.70 \\
\hline Septiembre & 8.50 & 7.70 \\
\hline Octubre & 6.10 & 7.60 \\
\hline & & \\
\hline
\end{tabular}

Fuente: Elaboración Propia. BCRP
El resultado de la Correlación, queda complementado con el coeficiente de determinación (R2) que como se aprecia en el Figura $\mathrm{N}^{\circ} 01$ es de $0.36 \%$, resultado que evidencia que las posiciones monetarias que tome el Banco Central solo explican en un $0.36 \%$ el comportamiento de las tasas de interés. Es decir existen factores ajenos a la oferta monetaria que influyen en la tasa de interés cuyo efecto llega el 99.64\%. Por este lado se puede evidenciar el débil anclaje del costo financiero a la política monetaria. Que en contrapeso está el grado de concentración y otros factores propios a las IFIS que explican la volatilidad de estas tasas, por ello, su ajuste debe estar condicionada a las acciones económicas y financieras que son propias de estas instituciones financieras. Como ya se señaló siendo una de las externalidades más importantes, las que se derivan del grado de cuasi monopolio. No hay que perder de vista que un solo banco maneja el 34\% de todas las operaciones del sistema y que 12 de estas organizaciones manejan portafolios que les permite poseer una cuota de par-

Figura No 01

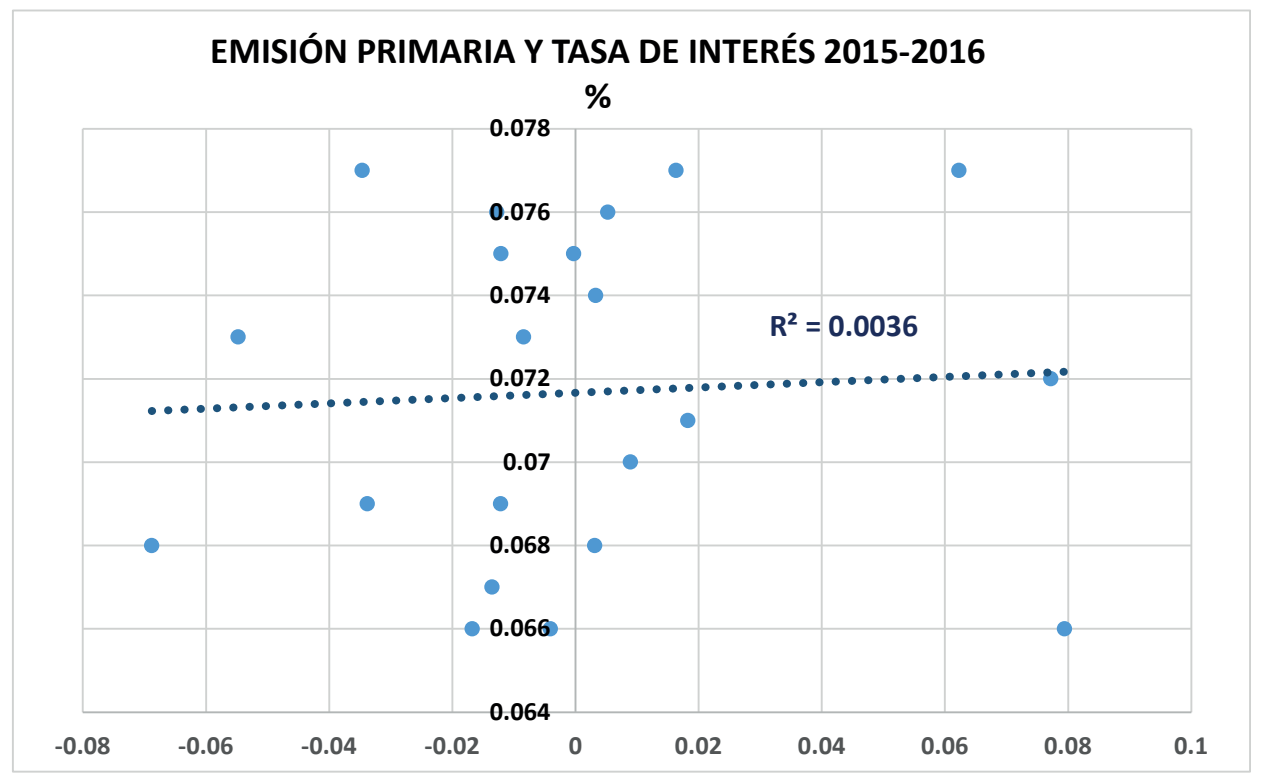

Fuente: BCRP, Elaboración propia. 
ticipación del 2\% del mercado.

Por otro lado, está la tasa de referencia que es una tasa interbancaria que es manejada por el Banco Central como parte de su Política Monetaria. Como dice la teoría macroeconómica, Sach (2011), en épocas de recesión y estancamiento el Banco debe relajar esta tasa, haciendo todo lo contrario cuando se llega a la zona de riesgo inflacionario. Asimismo se conoce que todas las tasas del Sistema Financiero deben seguir a la tasa monetaria, por lo que a través de una corrida de datos se puede afirmar que el grado de correlación debe acercarse a la unidad, de darse este resultado, las tasas activas como las pasivas tendrían el mimo recorrido en el tiempo, confirmándose un comportamiento asimétrico en el tiempo.

$\mathrm{Al}$ contrastar las cifras del BCR referente a estas dos variables, tasa de referencia y la de interés, se llega establecer para el periodo analizado $(2015$ - 2016) en forma mensual, el coeficiente de correlación (R) es del $0.93 \%$, cifra que refleja la alta relación de dependencia y de tipo directa entre estas tasas de interés. Para darle un mayor soporte a este resultado es preciso tomar en cuenta el coeficiente de determinación el cual llega al (R2) 0.87, es decir esta tasa llega a explicar en un $87 \%$ la formación de la tasa de interés. Comparativamente, esta última variable tendría una mayor influencia que la emisión primaria o inyección

Tabla No 02

Tasa monetaria y tasa de interés (\%)

\begin{tabular}{|l|c|c|}
\hline MES & TASA MONETARIA & TASA DE INTERÉS \\
\hline Enero & 3.25 & 7.10 \\
\hline Febrero & 3.25 & 6.90 \\
\hline Marzo & 3.25 & 6.80 \\
\hline Abril & 3.25 & 6.70 \\
\hline Mayo & 3.25 & 6.60 \\
\hline Uunio & 3.25 & 6.60 \\
\hline Julio & 3.25 & 6.60 \\
\hline Agosto & 3.25 & 6.80 \\
\hline Septiembre & 3.50 & 6.90 \\
\hline Octubre & 3.50 & 7.00 \\
\hline Noviembre & 3.50 & 7.10 \\
\hline Diciembre & 3.75 & 7.20 \\
\hline Enero & 4.00 & 7.30 \\
\hline
\end{tabular}

212/ QVIPURAMAYOC | Vol. 24(46) 2016

\begin{tabular}{|l|l|l|}
\hline Febrero & 4.25 & 7.30 \\
\hline Marzo & 4.25 & 7.40 \\
\hline Abril & 4.25 & 7.50 \\
\hline Mayo & 4.25 & 7.50 \\
\hline Junio & 4.25 & 7.60 \\
\hline Julio & 4.25 & 7.70 \\
\hline Agosto & 4.25 & 7.70 \\
\hline Septiembre & 4.25 & 7.70 \\
\hline Octubre & 4.25 & 7.60 \\
\hline
\end{tabular}

Fuente: Elaboración Propia. BCRP

Coeficiente de Correlación: $\mathrm{R}=0.93$.

Coeficiente de determinación $\mathrm{R}^{2}: 0.87$

de liquidez que realiza la autoridad monetaria en la formación de la tasa de interés.

La Figura $N^{\circ} 2$. Explica el comportamiento de estas dos tasas, como ya se señaló, presenta en su recorrido un comportamiento simétrico, es decir la tasa de interés por ser la variable dependiente está anclada a la tasa monetaria. Si se ha de elegir medidas para impulsar las inversiones vía la tasa de interés, la tasa monetaria tendría mayor impacto en este componente macroeconómico. No solo eso, también ejercería mayor dinamismo al consumo y la demanda agregada.

Como se dijo en un principio, para descontar el flujo de caja de las inversiones que conlleve a determinar los indicadores de rentabilidad como el VAN, es el costo promedio ponderado del capital, conocido más comúnmente como WACC, que ROSS (2010) lo define de la forma siguiente: "... es una tasa que pondera los costos financieros asumidos por financiar una inversión... "es así, y uno de los componentes es la tasa de interés. Si un proyecto de inversión se financia en el mercado bancario se asume una tasa activa o tasa efectiva activa TEA y si acude al mercado de capitales se tiene que pagar una tasa por la emisión de deuda.

Si bien es cierto que la tasa de interés depende de una serie de factores, como el riesgo que proyecta el sujeto prestatario, también, detrás de su comportamiento están las bondades o vicios del sistema de intermediación, como ya se evidencio, bajo este escenario, las medidas monetarias son poco relevantes para tal fin. Los bancos estructuran carteras que van desde créditos corporativos hasta hipotecarios inclu- 
Figura No 02

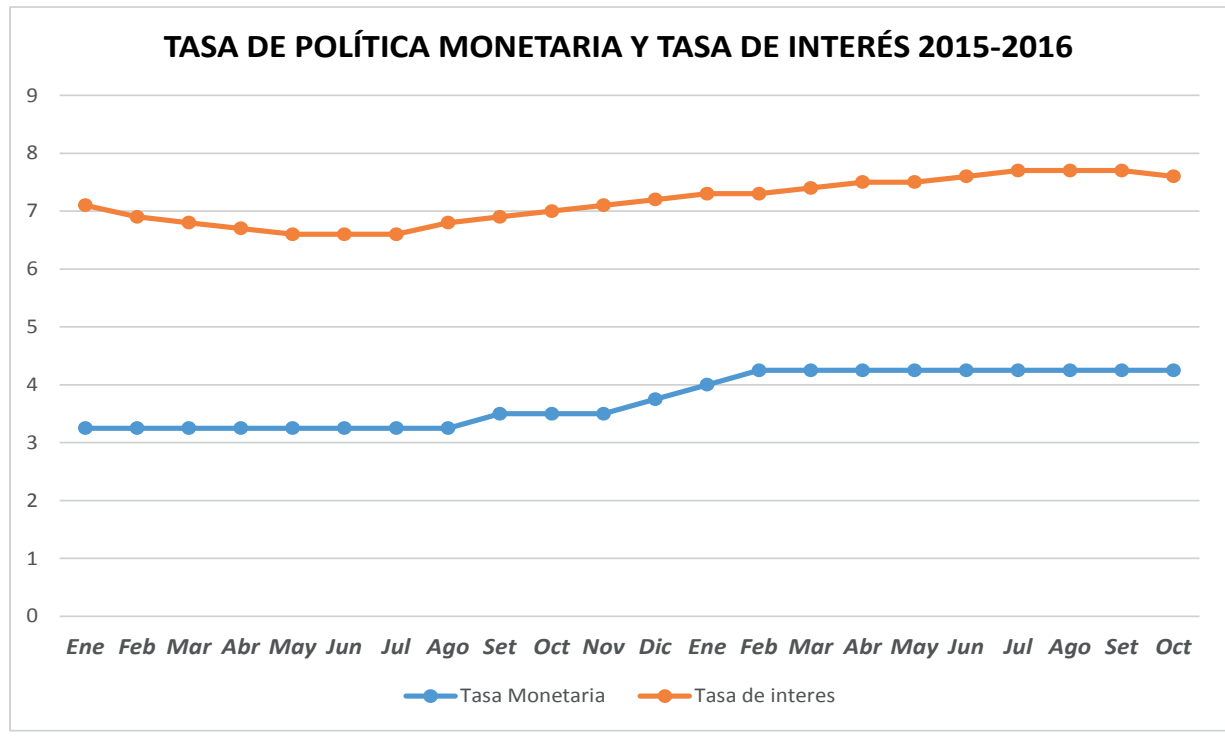

Fuente: BCRP, Elaboración propia.

yendo a las microempresas, la tasa asignada depende del perfil de cada uno de estos segmentos. La tasa de interés para créditos (TEA) más baja es cargada a los fondos que están dirigidos a financiar las inversiones del segmento corporativo que van del 5.5\% al 7\% y las MES (microempresa) en algunos casos llega al $35 \%$.

$$
\text { WACC }=\frac{D}{D+C}(\text { Tea }(1-t x))+\frac{C}{D+C}(\text { COK })
$$

En donde:

$\mathrm{D}$ : deuda

Tea : tasa efectiva activa ( tasa de interés que se coloca al sujeto prestatario)

Tx : impuesto a la renta

C: Capital de los accionistas o dueños de la inversión COK : costo financiero que se asigna al capital de los inversionistas, conocido como costo de oportunidad.

Se entiende que el WACC es la tasa mínima de rendimiento que se le pide a la inversión, el cual está en relación directa a la tasa de interés activa $\mathrm{y}$ al COK, por lo que se deduce que, de subir las tasas por cuestiones propias de los intermediarios financieros o por efectos de la política monetaria, los inversionistas deben de exigir mayor retorno a sus inversiones realizadas, con lo cual se castiga a estos flujos. Los cuales por su grado de sensibilidad pueden caer en escenarios de inflexión a una tendencia de crecimiento. Claro está, si se quiere dinamizar este componente de la demanda agregada, lo que queda es aplicar medidas expansionistas de tipo monetarias como es la de flexibilizar la tasa monetaria, bajar encaje o realizar operaciones de mercado abierto, pero siempre echando mano al monitoreo ex post de la medida para ver los grados de impactos y los retardos de las medidas.

Se señaló en un principio que para determinar la viabilidad de las inversiones se utiliza como indicador básico el Valor Actual Neto (VAN), cuya fórmula se detalla a continuación:

$$
\text { VAN }=-\mathbf{I}+\Sigma \frac{(\mathrm{Yt}-\mathrm{Et})}{(1+\text { WACC })^{\mathrm{t}}}
$$

Idenficandose las siguientes variables:

I : monto de la inversión

$\mathrm{Yt}=$ Ingresos generados por la inversión

Egresos generados por la inversión(costos y gastos)

WACC : Costo promedio ponderado del capital

Como se puede apreciar este indicador de rentabilidad esta en relación inversa a la tasa de descuento o WACC que a la ves esta última está afectada por 
la tasa de interés, que al someterlo al proceso de sensibilización con simulaciones al alza impactará negativamente en la rentabilidad de la inversión, El grado de impacto dependerá de cuan sensible sea las inversiones a los cambios del WACC y esta última a los de la TEA. Al entrecruzar el enfoque financiero con el de política monetaria y el de gestión bancaria se puede señalar que a través de estas tres vías se ve afectada la tasa de interés.

A través de un ejemplo se puede aclarar este último punto, a continuación se tiene el siguiente flujo de caja:

Se presenta una inversión de S/ 500 mil, de los cuales el $60 \%$ será financiado a través del banco y complementándose con recursos propios. La tasa

\begin{tabular}{|l|c|c|l|l|l|}
\hline $\mathbf{0}$ & 1 & 2 & 3 & 4 & 5 \\
\hline-500 & 150 & 200 & 280 & 300 & 320 \\
\hline
\end{tabular}

\begin{tabular}{|l|c|c|l|l|l|}
\hline Fuentes & Monto & Participa. & Tasa de interés & $(\mathbf{1}-\mathbf{T x})$ & Wacc \\
\hline Banco & 300 & 0.6 & $7 \%$ & 0.7 & 0.0294 \\
\hline Capital & 200 & 0.4 & $10 \%$ & & 0.04 \\
\hline Total & 500 & 1 & & WACC & $6.94 \%$ \\
\hline
\end{tabular}

\begin{tabular}{|l|l|}
\hline VAN & S/.502.28 \\
\hline
\end{tabular}

\begin{tabular}{|l|c|c|l|l|l|}
\hline Fuentes & Monto & Participa. & Tasa de interés & $(\mathbf{1 - T x})$ & Wacc \\
\hline Banco & 300 & 0.6 & 0.08 & 0.7 & 0.0336 \\
\hline Capital & 200 & 0.4 & 0.1 & & 0.04 \\
\hline Total & 500 & 1 & & Wacc & $7.36 \%$ \\
\hline
\end{tabular}

\begin{tabular}{|l|l|}
\hline VAN & S/.489.68 \\
\hline
\end{tabular}

efectiva que se aplica a la deuda bancaria es del $7 \%$ y el Costo de Oportunidad (COK) del 10\%. El impuesto a la renta es del $30 \%$

Como se puede apreciar en los cuadros anteriores, al cambiar la tasa de interés en un punto porcentual habrá un cambió en el WACC, el cual paso de $6,94 \%$ a $7.36 \%$ y con ello bajará la rentabilidad de las inversiones, ante un aumento en la tasa de interés en $1 \%$, el VAN cambia de S/502.28 a S/ 489.68, en $-2.51 \%$, demostrándose con esto la sensibilidad de las inversiones a la tasa de interés, que como se dijo es influenciada por el tipo de Política Monetaria y por las características oligopólicas o distorsiones que se puedan presentar en el mercado financiero de intermediación indirecta. Las cifras señalan que por cada punto que suba la tasa de interés, la rentabilidad de la inversión caerá en $2.51 \%$

\section{CONCLUSIONES}

1. Por las cifras señaladas, la tasa de interés, variable que influye en la determinación de la tasa de descuento (WACC) no es influenciada por la emisión primaria que es manejada por la autoridad monetaria. El coeficiente de determinación R2 del $0.36 \%$, explica la débil o casi nula influencia de los cambios en la oferta monetaria en la tasa de interés.

2. La tasa de interés está estrechamente correlacionada con la tasa de referencia o de política monetaria, el grado de simetría se ve evidenciada en el coeficiente de correlación el cual es del ( $R$ 
$=0.93)$. Esta tasa interbancaria ejerce mayor influencia en la tasa de interés activa y con ello en la tasa de descuento.

3. La eficacia de la política monetaria para impulsar las inversiones vía la tasa de interés, se ve limitada por las distorsiones que se presentan en el mercado financiero, específicamente el de intermediación indirecta, el cual presenta características oligopólicas.

\section{REFERENCIAS BIBLIOGRÁFICAS}

1. BACA,G. (2006). Evaluación de proyectos. Ediciones Mc Graw Hill. México. 391 ppp. Quinta edición

2. FISHER, D (2015): Macroeconomía Mac Graw Hill. México. Duodécima edición

3. SAPAG, N.(2008). Preparación y Evaluación de proyectos. Mac Graw Hill. México. 390 pp. Quinta edición

4. MANKIW( 2014) Macroeconomia- Antoni Bosch Editores. Barcelona. España. octava edición

5. ROSS, WESTERFIELD, JAFFE(2012) : Finanzas Corporativas . Edic. McGrawwHill. Mexico. Novena edición

6. Banco Central de Reserva (BCR) (2015). Memoria. Lima Perú

7. Banco Central de Reserva (BCR) (2015). Notas semanales. Lima Perú 